\title{
LAS TIC EN ESCUELAS RURALES: REALIDADES Y PROYECCIÓN PARA LA INTEGRACIÓN
}

\section{Resumen}

Fredy Yesid Mesa Jiménez

Doctor en Ciencias de la Educación Universidad Pedagógica y Tecnológica de Colombia (Tunja, Colombia)

fredy.mesa@uptc.edu.co

https://orcid.org/0000-0001-5962-0915

Artículo de Investigación

Recepción: 17 de diciembre de 2017 Aprobación: 23 de junio de 2018

DOI: https:/doi.org/10.19053/22160159.v9.n21.2018.8924

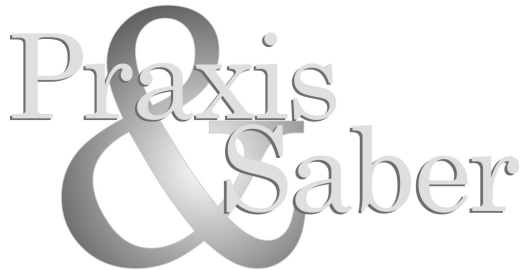

Revista de Investigación y Pedagogía Maestría en Educación. Uptc
Como baluarte de las políticas de Estado en materia de TIC, el gobierno ha ofrecido utilizar los medios tecnológicos en aras de potenciar el sistema educativo incluyente y de calidad, garantizando el acceso de todos los estudiantes del territorio nacional. Por ello, se hace necesario analizar, en el contexto rural del municipio de Tunja, el efecto de las políticas de Estado en materia de TIC, desde un abordaje relacionado con las facilidades o barreras para el acceso a la infraestructura computacional y comunicacional, el uso que los maestros han dado a los recursos con los cuales han podido contar en sus situaciones particulares y el grado de apropiación de estas tecnologías en su quehacer docente. En tal sentido, esta investigación de carácter mixto permitió develar las latentes necesidades y preocupaciones de los maestros. Además se concretó, a partir de la experiencia de los docentes, una secuencia para la incorporación de las TIC para los centros educativos rurales, que incluye la planificación, capacitación de maestros, disposición y gestión de recursos, mantenimiento y seguimiento e incentivos.

Palabras clave: TIC, educación, escuela rural, modelos de integración en TIC. 


\title{
ICT IN RuRAL Schools: ReAlities AND InTEgRATION PlanS
}

\begin{abstract}
The Government has offered to use technological means to strengthen a high-quality inclusive education system as a bastion of State policies on ICT, ensuring access for all students throughout the national territory. It is therefore necessary to analyze the impact of State policies on ICT in rural areas of the municipality of Tunja. To this end, facilities and barriers for access to communicational and computational infrastructure, the way teachers use the resources they have been given in specific situations, and the assimilation of these technologies in their pedagogical work are addressed. In that regard, this mixed methods research unveiled teachers' latent needs and concerns. Furthermore, based on their experiences, a process for the integration of ICT into rural schools was developed, which includes planning, teacher training, availability and management of resources, maintenance and tracking of incentives.
\end{abstract}

Keywords: ICT, education, rural school, ICT integration model.

\section{Les TiC DANS LES ÉCOlEs RuRALES: DES RÉalités et des Plans D’intégration}

\section{Résumé}

Le Gouvernement a proposé d'utiliser des moyens technologiques en vue de renfoncer un système éducatif inclusif et de qualité comme un bastion de ses politiques nationales en matière de TIC, en garantissant l'accès pour tous les élèves du territoire national. Par conséquent, il est nécessaire d'analyser l'impact des politiques des TIC dans le contexte rural de la municipalité de Tunja, en tenant compte des facilités et des obstacles à l'accès de l'infrastructure computationnelle et communicationnelle, de l'utilisation que les professeurs font des 
ressources qui leur sont donnés dans des situations particulières, et du degré d'appropriation de ces technologies à leur travail pédagogique. À cet égard, ce projet de recherche par des méthodes mixtes a dévoilé des préoccupations et des besoins latents des enseignants. En outre, sur la base de l'expérience des professeurs, un processus a été développé afin d'intégrer les TIC dans les écoles rurales, ce qui inclut la planification, la formation des enseignants, la disponibilité et la gestion des ressources, la maintenance et le suivi d'incitations.

Mots-clés: TIC, éducation, école rurale, modèle d'intégration des TIC.

\section{As Tic nas Escolas Rurais: Realidades e ProjeÇão para a INTEGração}

\section{Resumo}

Como um baluarte das políticas de Estado em matéria das TIC, o governo tem oferecido utilizar os meios tecnológicos a fim de potencializar o sistema de educação inclusiva e qualidade, garantindo o acesso de todos os alunos do território nacional. Por isto, é necessário analisar, no contexto rural do município de Tunja, o efeito das políticas de Estado na matéria das TIC, desde uma abordagem relacionada com as facilidades ou barreiras para o acesso à infraestrutura de computo e comunicação, o uso que os professores têm dado aos recursos com os quais têm podido contar em suas situações específicas e o nível de apropriação dessas tecnologias no seu trabalho de ensino. Neste sentido, esta pesquisa de caráter misto permitiu revelar as latentes necessidades e preocupações dos professores. Ademais se concretou, a partir da experiência dos professores, uma sequência para a incorporação das TIC para as escolas rurais, incluindo o planejamento, formação de professores, disposição e gestão de recursos, manutenção, monitoramento e incentivos.

Palavras-chave: TIC, educação, escola rural, modelos de integração nas TIC. 


\section{Introducción ${ }^{1}$}

"La escuela no permanece ajena a los impulsos activos de una sociedad que, pese a las alarmantes diferencias en el grado de desarrollo según la localización geográfica, alienta con fuerza

la «migración digital»”. (San Martín, 2009, p. 33)

El gobierno nacional, a través del Ministerio de Tecnologías de la Información y la Comunicación [MinTIC] y programas como Computadores para Educar, Kiosco Vive Digital, entre otras, ha buscado dotar de recursos y estrategias tecnológicas a las instituciones educativas de contextos rurales, en algunos casos con acompañamiento a docentes, a cargo de gestores del proceso. No obstante, así como en algunos países de América Latina, también en Colombia existen dificultades en la incorporación de las tecnologías en los contextos educativos. Una de ellas se relaciona con el uso de computadoras en áreas urbanas y rurales. Mientras que en las zonas urbanas el 69\% de los jóvenes usa computadora, en las áreas rurales solo lo hace el 36,7\%, develando una disparidad considerable (IPEE Unesco, 2014), además del hecho de que los procesos de planificación con TIC en las ruralidades poco dan cuenta de la heterogeneidad de las comunidades, las necesidades del entorno y el desarrollo productivo laboral (Camarda, 2016).

Situando en contexto esta realidad, según el acercamiento a las diez instituciones educativas rurales del municipio de Tunja, estos centros educativos se caracterizan por recibir en sus aulas estudiantes que provienen de familias de bajos recursos, que hacen poco probable la prevalencia de dispositivos tecnológicos como computadores, tabletas y acceso a internet en sus hogares. Dicha situación hace evidente la brecha digital, pese a ser muy cercanos a la capital del departamento de Boyacá. Así, se evidencian dificultades sustanciales en el acceso a las TIC fuera de los espacios escolares.

1 El presente trabajo se presenta como parte de los resultados del proyecto de investigación denominado Diseño de un modelo para la incoporación de las TIC, SGI 2076, UPTC, adelantado por Luis Molina, joven investigador del Grupo de Investigación Historia y Prospectiva de la Universidad Latinoamericana (HISULA) de la línea de Investigación Universidad Formación de Docentes e Interculturalidad. 
En este sentido, la única manera de acceso a las TIC para muchos de estos estudiantes lo representan las prácticas de aula. Por lo tanto, el estudio pretendió identificar la disponibilidad de recursos tecnológicos en estas instituciones educativas, la importancia que presentan para el desarrollo de las actividades curriculares y la existencia de planes estratégicos o modelos para la incorporación educativa de las TIC.

El estudio se centró en las sedes rurales de instituciones oficiales de la ciudad de Tunja, que corresponden a la Institución Educativa Rural del Sur, la cual cuenta con 7 sedes: Barón Gallero, Barón Germania, Chorro Blanco, Francisco de Paula Santander, José Joaquín Castro Martínez, Runta Abajo y Runta Arriba; y la Institución Educativa Gustavo Rojas Pinilla, en 3 sedes, La Esperanza, El Porvenir y Florencia. Estas instituciones se encuentran localizadas al sur y al occidente de la ciudad de Tunja.

En estas sedes, al 55\% de los docentes se les aplicó una encuesta que previamente fue validada por expertos. Su sistematización y análisis permitieron evidenciar las dificultades que las instituciones presentan en términos de conectividad e infraestructura tecnológica; así como la necesidad de estrategias de capacitación y transferencia tecnológica, además de un plan estratégico para la incorporación de TIC, como se especifica en el apartado de resultados.

\section{La dimensión política de la incorporación de las TIC en las instituciones educativas colombianas}

Las primeras iniciativas destinadas a incorporar las TIC aparecen a partir de los años ochenta, las cuales afectaron la educación básica, media y superior (Mesa, 2012; Mesa \& Forero, 2016; Soto, Mesa \& Caro, 2012). De esta manera, en 1994 se publicó la Politica Nacional de Cienciay Tecnología 1994-1998 con el propósito de

(a) desarrollar en el país una capacidad para utilizar la informática y los computadores en la educación y la ciencia; (b) facilitar el acceso a información tanto a través de redes nacionales como de redes internacionales y; (c) promover el desarrollo de estándares que faciliten la integración en informática. (DNP, 1994, p. 13). 
El desarrollo de las telecomunicaciones se plantea seguidamente en el Plan Nacional de Desarrollo 1998-2002 (Colombia, 1998) y es en 1999 en que se inicia el programa que propendió por el uso de los teléfonos comunitarios en aquellas localidades rurales, denominado Programa Compartel. Así mismo, Computadores Para Educar se plantea en el documento Conpes 3063 de 1999, como un programa de donación masiva de computadores (DNP, MEN \& SENA, 1999), que benefició a instituciones educativas públicas, normales superiores, casas de la cultura y bibliotecas.

No obstante, como afirman Barón \& Gómez (2012), estas iniciativas no lograron satisfacer los procesos de desarrollo:

Hasta ese momento la acción estatal se había centrado en la mejoría de los índices de cubrimiento de la educación y de penetración de las TIC, mediante el desarrollo de infraestructura y equipamiento, dando mayor importancia a los discursos técnicos y haciendo más difícil adelantar dinámicas participativas. (p. 44)

De esta forma, el desarrollo de infraestructura y equipamiento, que hasta entonces no había sido suficiente para garantizar la participación dinámica de las comunidades, comenzó a replantearse a comienzos del siglo XXI, por tal motivo se modifican algunos programas relacionados a la Agenda de Conectividad, como lo fue Computadores para Educar², indicando como obligación del Ministerio de Educación, entre otras, garantizar la "apropiación efectiva de la tecnología por parte de las instituciones públicas beneficiadas de los equipos" (Ministerio de Comunicaciones, 2000, p. 4).

En la primera década del nuevo siglo, se reformulan otros, como Compartel, y se crean nuevos lineamientos de la política de telecomunicaciones. En 2008 se publica el Plan Nacional de TIC En linea con el futuro 2008-2019', que tiene como misión "la inclusión social y la competitividad del país a través de la apropiación y el uso

2 Según el Informe de Gestión de Computadores para Educar 2016, al menos 72.529 docentes y directivos docentes culminaron satisfactoriamente el proceso de formación en el uso pedagógico de las TIC, se demanufacturaron 24.381 equipos de cómputo, equivalente a cerca de 500 toneladas de residuos electrónicos en procura de la sostenibilidad ambiental y se continuó con la entrega de terminales, destinando así 194 mil a estudiantes y 65 mil para uso de docentes (MEN, MinTICSENA, \& Computadores para Educar, 2016). 
adecuado de las TIC, tanto en la vida cotidiana como productiva de los ciudadanos, las empresas, la academia y el Gobierno." (Ministerio de Comunicaciones, 2008, p. 9).

Para 2009, con el propósito de masificar el uso de las TIC e impulsar el uso eficiente de la infraestructura, el Ministerio de Comunicaciones se convierte en Ministerio de Tecnologías de la Información y la Comunicación [MinTIC]. Desde allí se han impulsado iniciativas como kioscos digitales, fortalecimiento de las bibliotecas como puntos clave para el acceso público a TIC, puntos Vive Digital, zonas wifi, estrategias para la denuncia de contenidos ilegales como el abuso sexual, la explotación sexual comercial y la pornografía infantil y adolescente, entre otros.

Estos programas han logrado dotar de recursos y estrategias tecnológicas a las instituciones educativas rurales del país. No obstante, dichas políticas no han sido suficientes, como lo afirman algunos autores como Felizzola (2010), quien afirma que "las tecnologías tienen un lenguaje estilizado y el diseño de los programas, procesos y procedimientos de acceso y retribución, tienen un carácter casi exclusivamente urbano" (p. 102), que no responde a las características de los contextos de manera que permita el empoderamiento de estas comunidades a través del acceso a la información.

Además, estas iniciativas en las zonas rurales suelen significar altos costos de operación, por tratarse de instituciones apartadas, lo cual da como resultado la generación de altas expectativas, pero en términos de funcionamiento los proyectos solo son sostenibles por cortos periodo de tiempo (Ortiz, 2005; Felizzola, 2010) o dependientes de los propósitos políticos de los dirigentes del momento.

Para San Martín (2009), una de las dificultades de la incorporación de las TIC en los contextos educativos gravita en la brecha digital endógena, teniendo en cuenta que los usuarios carecen de un conocimiento claro de los aparatos tecnológicos que pretenden usar. $\mathrm{Al}$ respecto añade:

No se trata de convertir a los alumnos en expertos conocedores de los entresijos tecnológicos, ni mucho menos. Simplemente se les debería facilitar herramientas conceptuales para que entiendan y desmitifiquen por qué sucede lo que sucede o por qué son 
capaces de hacer lo que hacen las máquinas electrónicas [...] así como consecuencias colaterales que se desprendan de su uso [...] La dificultad, sin embargo, estriba no solo en alcanzar cierto grado de comprensión de los procesos, sino que al intensificarse de modo rutinario la interacción con la máquina, se vacía de contenido cultural. (pp. 51-52)

En las aulas, la instrumentalización de las TIC obvia la integración desde lo pedagógico, lo que ubica a la escuela en el reto de transformar las prácticas con miras a "formar a la ciudadanía en un pensamiento fundamentado y democrático ante el desarrollo tecnocientífico" (San Martín, 2009, p. 53), idea que comparte Mattelart (2007) cuando indica que

la falta de una propedéutica de la apropiación de las tecnologías digitales anda lado a lado con la fascinación por el objeto técnico y la carencia de una reflexión sobre la historia de utopía pedagógica que no esperó las nuevas tecnologías de la comunicación y de multimedia [...] reflexionar sobre el múltiple entrecruzamiento de las mediaciones sociales, culturales y educativas por las cuales se construyen los usos del mundo digital y que están en el propio origen de la vida democrática. (p. 176)

Se trata no solo de pensar en una escuela dotada de tecnologías digitales, sino de una escuela que piense críticamente el uso y apropiación de los recursos, desde el maestro que sabe cuándo, cómo y dónde es propicio desarrollar una clase a partir de la mediación de las TIC y cuándo su uso no resulta imperativo; hasta el estudiante que cuestiona el impacto que generan en su desarrollo y participación.

\section{Modelos de integración curricular de las TIC}

Según las Competencias TIC para el desarrollo profesional Docente (MEN, 2013a), el docente es un agente integrador cuando de manera autónoma y creativa usa las TIC, descubriendo el potencial que significan para la planeación, evaluación y el desarrollo de las prácticas de aula. De igual manera, el docente integrador de las TIC aprovecha los recursos disponibles en la web para su formación continua y asume de manera crítica las implicaciones sociales que su inclusión representa.

Para alcanzar el propósito de la integración, se han planteado diversos modelos que involucran etapas o fases, proponiendo un camino que 
el maestro y la institución educativa puede organizar para la ejecución de dicha empresa.

Bacigalupo \& Montaño (2005) enuncian seis fases para la incorporación de TIC en el proceso de innovación docente, a saber: fase 0: involucra la reparación y caracterización del entorno, y definición de la construcción de procesos; fase 1: sensibilización de la innovación a través de la definición de los involucrados y socialización de temáticas innovadoras vigentes; fase 2: exploración de recursos de manera que se busque el uso efectivo de los materiales tecnológicos -material digital, plataforma-; fase 3: contempla la exploración de recursos pedagógicos; fase 4: generación de recursos, mediante el establecimiento de mecanismos de trabajo formal y por proyectos que permita la interdisciplinariedad de los recursos; y finalmente en la fase 5: integración de recursos en el proceso de enseñanza y aprendizaje, evidenciado en los planes de estudio.

Por su parte, Londoño \& Castillo (2012) en la primera etapa proponen la definición del estado deseado que permitirá direccionar las actividades en un determinado periodo. Para ello se hace precisa la revisión de los procesos de enseñanza y aprendizaje. La segunda etapa es la de planificación, la cual busca el aprovechamiento de los recursos $\mathrm{y}$ condiciones disponibles por la institución, lo que implica el diseño y gestión de estrategias. En la tercera etapa se procede a la implementación, lo que supone considerar actividades, recursos, tiempos y responsables. De manera trasversal a las etapas anteriormente descritas, los autores proponen los componentes de evaluación y seguimiento.

En la investigación titulada Desarrollo de una metodología para integrar las TIC en las IE de Montería, Soto, Franco \& Giraldo (2014) discriminan cuatro momentos: inicialmente un diagnóstico y priorización de la problemática TIC; el momento normativo, que contempla el diseño del plan bajo objetivos e indicadores claros y alcanzables; el momento estratégico, el cual involucra aspectos como la formación, inclusión de las TIC en el currículo, administración y gestión de la infraestructura y evaluación de contenidos; en el momento táctico-práctico se ejecutan los objetivos e indicadores y se redefine el plan según las circunstancias. Para cada momento se ha de incorporar un proceso evaluativo que garantice la estabilidad, valoración y realimentación. 
López (2015) plantea cinco ejes para la integración de TIC al currículo, descritos de la siguiente manera: 1) dirección institucional, que hace referencia al liderazgo de las directivas administrativas, necesario para adelantar los cambios en infraestructura y cultura organizacional; 2) infraestructura TIC, que atiende al hardware, software, conectividad y soporte técnico; 3) coordinación y docente TIC, este eje hace énfasis a las funciones de la institución, el coordinador informático y el equipo docente; 4) docentes de otras áreas, que se refiere al trabajo interdisciplinar; y 5) recursos digitales, como el eje que garantiza la disponibilidad de herramientas informáticas y contenidos digitales.

El Banco Mundial, desde el programa World Links, propone una aproximación sistemática para el uso de las TIC en las salas de clase con el propósito de desarrollar competencias del siglo XXI, tanto en estudiantes como en profesores. Las acciones propuestas se desglosan en seis niveles, así:

(1) ministerios de educación para el desarrollo de un plan estratégico de TIC, (2) desarrollo profesional del profesor, (3) impacto en el estudiante mediante las metodologías para el uso de TIC en las que se capacita a los profesores (4) medidas de generación de recursos para la sustentabilidad de las tecnologías en los establecimientos educativos, (5) implementación de iniciativas de monitoreo y evaluación, (6) desarrollo de capacidad local para construir organizaciones locales que ayuden al Ministerio a expandir, mantener y monitorear los programas TIC, permitiendo la sustentabilidad regional y nacional. (Claro, 2010, p. 11)

Finalmente, Pérez, Fandos \& Aguaded (2009) plantean los obstáculos que se pueden presentar para el desarrollo de los proyectos en TIC y que deben preverse en la construcción de un modelo para su integración. Entre ellos se encuentran la ausencia de incentivos, la inflexibilidad del proyecto, la inestabilidad de plantillas o formatos de seguimiento, gestión centralizada del sistema, la ausencia de un coordinador y carencias en la atención de la administración.

De esta manera, cada modelo plantea una serie de acciones o etapas que es posible tener en cuenta en el propósito de integrar las TIC a los procesos institucionales, dependiendo de los contextos que suponen tener en cuenta la disponibilidad de recursos y la proyección de adquisición de los mismos, así como lo relacionado al recurso humano -líderes, capacitaciones e incentivos-. 


\section{Perspectiva metodológica}

La investigación se desarrolló con el objetivo de caracterizar la manera en que las instituciones educativas del contexto rural del municipio de Tunja han experimentado la integración de las TIC en el quehacer del maestro y en el desarrollo del currículo.

La investigación se realizó bajo el enfoque mixto, en el cual se utilizó mayormente información cuantificable para develar la realidad sobre el fenómeno que se estudió. Para tal caso, se tomó una muestra de 36 docentes -el 55\% de los profesores de la instituciones rurales de Tunja- a quienes se les hizo una encuesta - de preguntas cerradas y abiertas- que contempló tres aspectos de la integración de las TIC, surgidos de la revisión literaria: 1) recursos tecnológicos existentes en las instituciones, 2) las TIC en el currículo y uso en la práctica educativa y 3) planificación de las TIC y visión estratégica.

En este orden, la investigación se desarrolló a partir de etapas o momentos, de la siguiente manera:

Primera fase: se realizó una fase conceptual y de revisión documental que permitió caracterizar modelos de integración curricular de las TIC y analizar desde las políticas un panorama de la situación actual de las TIC en Colombia. También permitió la establecer los aspectos de análisis de la investigación.

Segundafase: comprendió el acercamiento al contexto desde la formulación y aplicación de la encuesta, que previamente fue diseñada y validada a través de un grupo de expertos ${ }^{3}$. De esta manera fue posible recolectar información primaria.

Tercera fase: se constituyó de la sistematización de la información, para lo cual se analizaron las variables de manera independiente a través de su frecuencia-recursos tecnológicos existentes en las instituciones, las TIC en el currículo y uso en la práctica educativa y planificación de las TIC y visión estratégica-, con apoyo de las opiniones y argumentos realizados

3 Se realizó a través de la valiosa ayuda de profesores de la Licenciatura en Informática y Tecnología de la UPTC. Se analizaron las observaciones y sugerencias que permitieron ajustar el instrumento aplicado. 
por los encuestados en las preguntas abiertas, sobre el contexto y las maneras en que las instituciones educativas del sector rural de Tunja han venido integrando las mediaciones tecnológicas.

Finalmente se devela la secuencia para el plan estratégico de incorporación de TIC indicada por los docentes de las escuelas rurales de Tunja.

\section{Resultados}

En la encuesta aplicada al 55\% de los docentes de las instituciones educativas de Tunja ubicadas en el sector rural, se indagó por: 1) recursos tecnológicos existentes en las instituciones, 2) Las TIC en el currículo y uso en la práctica educativa y 3) planificación de las TIC y visión estratégica. A continuación se presentan los principales resultados.

\section{Recursos tecnológicos existentes en las instituciones analizadas.}

Uno de los aspectos para la indagación fue la existencia de conectividad, para lo cual se preguntó por el tipo de conexión de internet y la calidad percibida del servicio, esto teniendo en cuenta que una de las etapas o acciones en un modelo de integración de TIC, según lo reporta la revisión realizada, contempla lo relacionado a hardware, software $\mathrm{y}$ conectividad como eslabón importante.

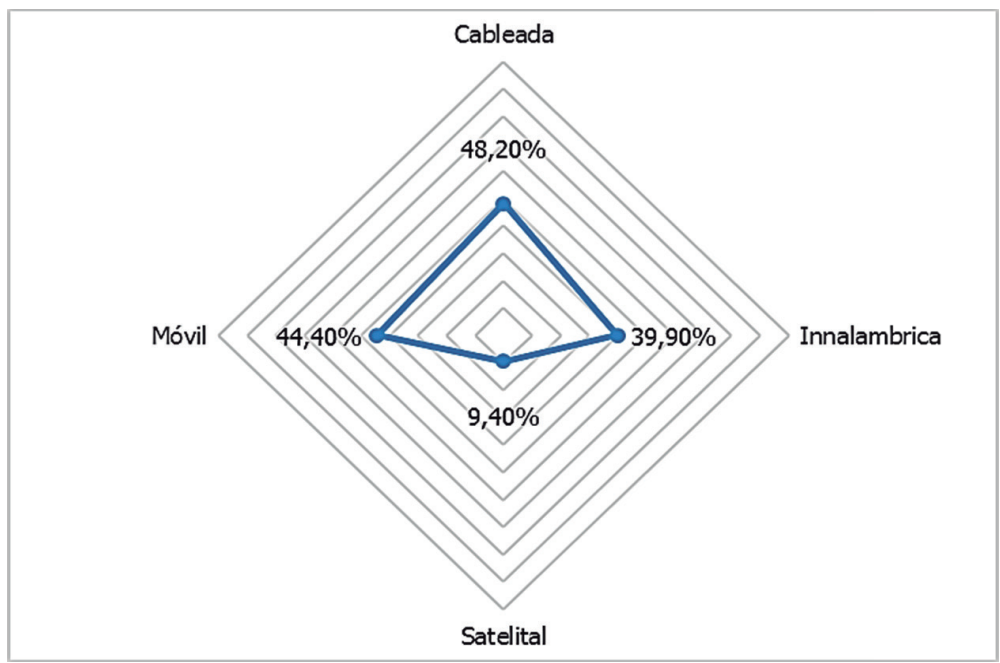

Figura 1. Conectividad en las I.E.

Fuente: elaboración propia. 
En este sentido, se encontró que el 52,8\% de los docentes afirman no contar con conexión por cable a internet. Del 48,2\% de los docentes restantes que afirman disponer de conectividad por cable, el 25\% la considera regular y solo un 2,8\% -un docente-lo considera muy bueno. En cuanto a la conexión inalámbrica, el 61,1 \% de los encuestados indica que no cuentan con conexión inalámbrica en la institución educativa, mientras el 39,9\% que indica tener acceso a conectividad inalámbrica la califica como: regular $-13,99 \%-$, muy buena $-5,6 \%-$, excelente $-2,8 \%$ - Solo un 9, 4\% de los docentes afirma contar con internet satelital en sus instituciones, pero para ninguno de ellos califica esta conexión como excelente.

El 44,4\% de los docentes encuestados afirma utilizar sus datos móviles para el desarrollo de las actividades académicas, lo cual permite evidenciar el compromiso individual de los maestros por llevar a cabo la integración de las TIC a sus prácticas, pese a las condiciones que afrontan en las instituciones educativas. En concordancia con Menchén (2009), "los escenarios de la sociedad del futuro están sufriendo continuos cambios y los maestros también están afectados por esta tendencia universal. Las competencias del docente han cambiado, no basta con ser maestro, hay que ser además creativo” (p. 279).

De igual forma, se preguntó a los docentes si consideraba que podían hacer uso de las TIC en sus clases sin contar con el servicio de internet, a lo cual un $83,3 \%$ indico que sí, teniendo en cuenta que:

-El docente puede crear las actividades en su casa y traerlas en CD o memoria para desarrollar en la escuela.

-Constantemente hay fallas de energía eléctrica y los docentes deben ser recursivos para trabajar con los niños y además traer las tabletas cargadas de nuestras casas.

-Se realizan actividades previamente descargadas y algunas que no requieren de internet.

-Se puede hacer uso de algunos aparatos como videobeam y llevar un material audiovisual ya preparado para presentar dentro del aula -El internet no es la base y se puede trabajar herramientas offline y descargar contenido (Información de encuesta con pregunta abierta. [2 de marzo de 2018] Encuestador: Luis Eduardo Molina. Tunja) 
En contraste, aquellos docentes que indicaron que no era posible hacer uso de las TIC por carencia de internet, los argumentos estuvieron relacionados con el desconocimiento de otras maneras de hacer uso de estas tecnologías independientes de la conectividad o por considerar complejo el acceso a recursos educativos sin este servicio.

De igual manera, se indagó por el número de estudiantes por computador y por tableta. Se encontró que la razón estudiante-tableta está en aproximadamente 3,7 estudiantes por tableta y 3,5 estudiantes por computador, es decir para cada 7 estudiantes hay 2 tabletas y para cada 37 estudiantes hay 10 computadores. Sin embargo, al omitir un dato extremo en el cual hay una tableta para cada 30 estudiantes, en promedio por cada 5 estudiantes hay una tableta. De otro lado, 5 docentes desconocen la razón estudiante por computador, y 13 desconocen la razón estudiante por tableta.

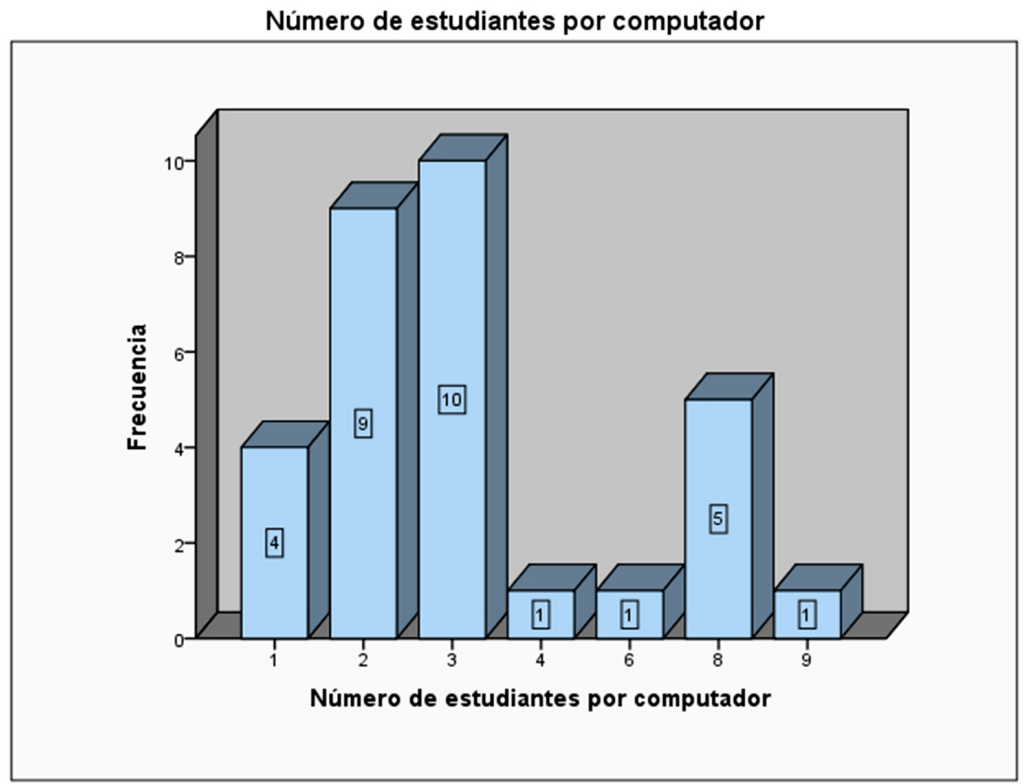

Figura 2. Estudiantes por computador.

Fuente: elaboración propia. 
Número de estudiantes por Tablets

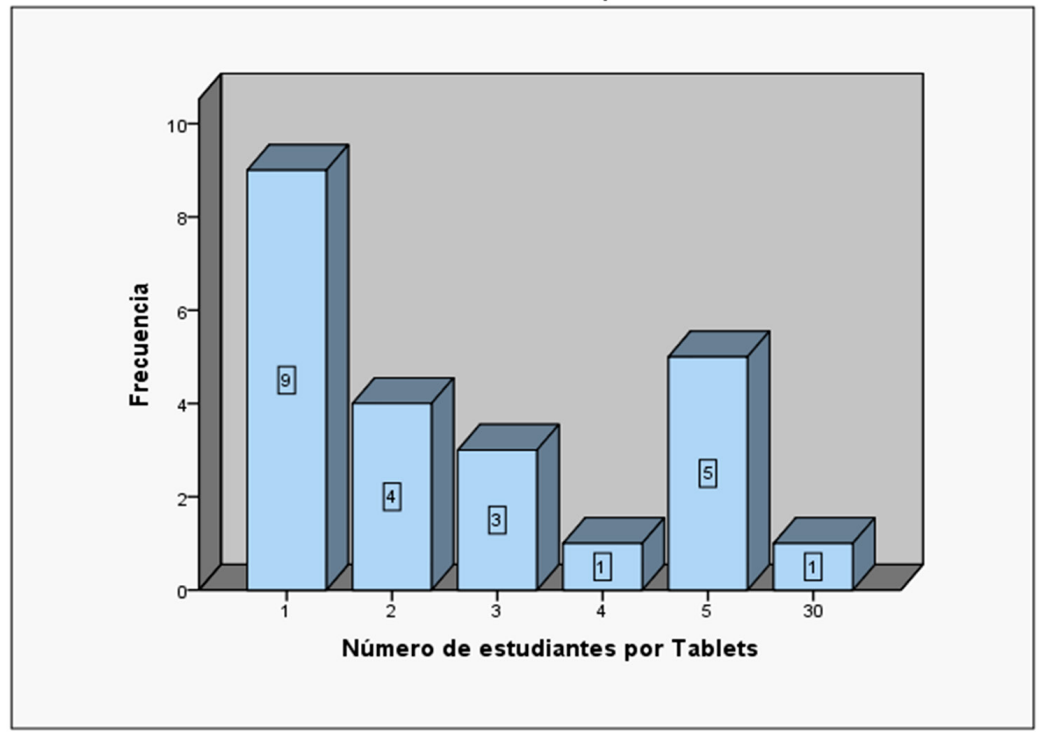

Figura 3. Número de estudiantes por tableta.

Fuente: elaboración propia.

En relación con el mantenimiento de las herramientas tecnológicas, un $63,9 \%$ indica que sí se realiza a los computadores, portátiles y medios audiovisuales, no obstante, es muy esporádica, generalmente de forma anual; mientras que un 33,3\% respondió que no reciben ningún tipo de mantenimiento.

De esta manera, es posible pensar en la necesidad de estrategias de capacitación y transferencia tecnológica que le permitan al maestro dar sostenibilidad a los proyectos basados en TIC a través de acciones de mantenimiento preventivo y, dentro de lo posible, también correctivo a los recursos de los cuales disponga la institución, llámese tabletas, computadores de mesa o portátiles. Esta estrategia ha resultado eficaz en la implementación y continuidad de proyectos en áreas rurales, donde los mismos sujetos empoderados se hacen cargo de sus recursos (Morante, Mocelin \& Zilles, 2006).

\section{Las TIC en el currículo y uso en la práctica educativa.}

En cuanto a la importancia que tienen las TIC dentro del currículo, se encontró: 
Tabla 1

Importancia de las TIC en el currículo

\begin{tabular}{ccccc}
\hline & Frecuencia & Porcentaje & Porcentaje válido & Porcentaje acumulado \\
\hline Alta & 11 & $30,6 \%$ & 30,6 & 30,6 \\
Media & 19 & $52,8 \%$ & 52,8 & 83,3 \\
Baja & 6 & $16,7 \%$ & 16,7 & 100 \\
\hline Total & 36 & $100 \%$ & 100 & \\
\hline
\end{tabular}

Fuente: elaboración propia.

El 30,6\% de los docentes considera que es alta, el 52,8\% considera que es media y un 16,7\% que es baja. En cuanto a las áreas en las cuales los docentes consideran que las TIC se utilizan en mayor medida, se reporta lo descrito en la tabla 2, dando mayor puntuación a lenguaje, matemáticas y ciencias naturales.

\section{Tabla 2}

Área en la cual se utilizan las TIC en mayor medida

\begin{tabular}{cccc}
\hline \multirow{2}{*}{ Área } & \multicolumn{2}{c}{ Respuestas } & $\begin{array}{c}\text { Porcentaje según los } \\
\text { docente }^{*}\end{array}$ \\
\cline { 2 - 3 } N & Porcentaje & $62,90 \%$ \\
Lenguaje & 22 & $12,60 \%$ & $65,70 \%$ \\
Matemáticas & 23 & $13,20 \%$ & $74,30 \%$ \\
Ciencias Naturales & 26 & $14,90 \%$ & $60,00 \%$ \\
Sociales & 21 & $12,10 \%$ & $65,70 \%$ \\
Inglés & 23 & $13,20 \%$ & $71,40 \%$ \\
Informática & 25 & $14,40 \%$ & $14,30 \%$ \\
Educación Física & 5 & $2,90 \%$ & $25,70 \%$ \\
Ética & 9 & $5,20 \%$ & $31,40 \%$ \\
Religión & 11 & $6,30 \%$ & $25,70 \%$ \\
Arte & 9 & $5,20 \%$ & $497,10 \%$ \\
\hline Total & 174 & $100,00 \%$ &
\end{tabular}

Fuente: elaboración propia.

\section{Planificación de las TIC y visión estratégica.}

Se preguntó a los docentes si en la institución educativa se realizaba algún seguimiento al uso de las TIC, a lo cual el 66,7\% manifestó no conocer algún proceso relacionado. En relación con la pregunta sobre

* El porcentaje de respuestas en relación con los profesores docentes supera el 100\% debido a que cada profesor podía escoger una o varias asignaturas. 
si consideraba que los recursos tecnológicos con los cuales la institución cuenta responden a las políticas de Estado en materia de TIC, el 72,2\% concordó que no, argumentando, en términos generales que persiste la desactualización de los equipos, la no continuidad ni seguimiento a las políticas de Estado, además de ser políticas ambiciosas sin tener en cuenta la realidad de los contextos.

El Consejo Latinoamericano de Ciencias Sociales [Clacso] concuerda con las posturas de los maestros, entre tanto considera a los entes estatales como agentes que desconocen las particularidades de las regiones junto con su gente, de igual manera la riqueza de las experiencias de los maestros derivadas de su relación con los estudiantes, con otros colegas y con la comunidad en general (Clacso, 2015).

Además, los docentes indican que el gobierno no invierte en capacitaciones de calidad para maestros. En relación con este último argumento, también se indagó sobre la participación en cursos de formación en materia de TIC, a lo cual el 77,8\% de los docentes encuestados contestó afirmativamente, mientras un 19,4\% indicó no haber tomado este tipo de cualificaciones.

Lo anterior permite evidenciar que, si bien los docentes participan y no desconocen los esfuerzos de las políticas de Estado por ofrecer actividades de capacitación, cuestionan la calidad de las mismas. Según la Unesco

la dificultad, ya reconocida, que tienen los profesores para asumir reformas en su práctica docente demuestra que quienes las diseñan no toman nota que el cambio docente es un proceso de aprendizaje y que como tal se construye sobre una realidad ya enraizada en la práctica cotidiana de cada maestro. (Unesco, 2002, p. 36)

En esta lógica, las políticas y procesos de formación continuada de docentes en materia de TIC no deben buscar reemplazar una práctica por otra que sea mediada por las TIC, sino dar respuesta o ser la consecuencia razonable de lo que ha ido descubriendo en su ejercicio docente.

Finalmente, extraídos de las investigaciones realizadas por diversos autores (Londoño \& Castillo, 2012; Soto, Franco \& Giraldo, 2014; Vásquez, 2005), se sugirieron cinco procesos para una secuencia de consolidación de un entorno de aprendizaje que favorezca la incorporación de las TIC 
en las instituciones rurales, a saber: 1) planificación, 2) capacitación de maestros, 3) recursos tecnológicos, 4) mantenimiento y seguimiento de los recursos tecnológicos y 5) programas de incentivos. Los procesos se dejaron abiertos para que los docentes propusieran otros componentes que considerasen importantes. En este sentido, se pidió organizar la secuencia para determinar etapas de un plan estratégico para la incorporación educativa de las TIC en instituciones rurales, obteniendo como resultado lo expuesto en la siguiente tabla.

Tabla 3

\begin{tabular}{ccc}
\multicolumn{3}{c}{ Secuencia para plan estratégico } \\
\hline Etapa & Actividad & Porcentaje \\
\hline Primera Etapa & Planificación & 47,1 \\
Segunda Etapa & Capacitación de Maestros & 41,2 \\
Tercera Etapa & Recursos tecnológicos & 38,2 \\
Cuarta Etapa & Mantenimiento y seguimiento de & $35 \%$ \\
Quinta Etapa & los recursos tecnológicos & $67,6 \%$ \\
\hline
\end{tabular}

Fuente: elaboración propia.

Cabe señalar que los docentes no añadieron otros componentes diferentes a los propuestos. De esta manera, la secuencia para el plan estratégico de incorporación de TIC señalado por los docentes de las escuelas rurales se compone de la siguiente manera

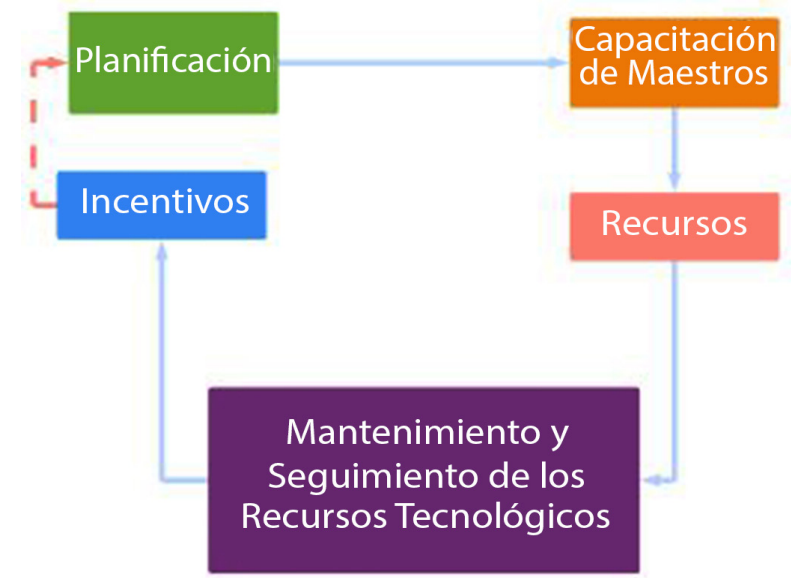

Figura 4. Propuesta de secuencia para la incorporación de TIC en las escuelas rurales de Tunja.

Fuente: elaboración propia. 
Planificación: En esta etapa es prudente considerar las realidades institucionales, es decir, los aspectos de fragmentación o rigidez curricular, adopciones tecnófilas o tecnofóbicas ${ }^{4}$ de parte de docentes, y la revisión de herramientas de control para validar acciones y recibir realimentación (Hernández, Gómez \& Montenegro, 2014).

Capacitación de maestros: Según la Unesco (2004), los cursos dictados en centros de capacitación o universidades en los cuales se enseña a manejar ciertas aplicaciones específicas de software o hardware son difíciles de aplicar en la práctica, además por no contar con mecanismos de perfeccionamiento ulteriores y apoyo continuo. De esta manera se propone, ofrecer las capacitaciones a maestros en la propia institución educativa para abordar las parvedades específicas que tenga cada docente en materia de uso de TIC.

En un programa en el Reino Unido se capacitó a asesores docentes, brindándoles recursos tecnológicos y la oportunidad de trabajar junto a los profesores dentro de sus clases. Los asesores podían observar el contexto en que los profesores trabajaban y, junto con ellos, desarrollar una forma apropiada de incorporar las TIC a su plan de estudios y de promover una organización efectiva de los recursos tanto dentro de la institución como en otras instituciones educativas de la región. Este enfoque, sin embargo, requiere una inversión importante. (Unesco, 2004)

Recursos tecnológicos: Las herramientas TIC, tales como portátiles, computadores de mesa, proyectores, televisores, micrófonos, audífonos, conectividad, recursos digitales y conectividad, son esenciales para poder garantizar la integración de las TIC al currículo, desde el desarrollo de competencias relacionadas al uso de las tecnologías y otras competencias relacionadas a la selección y el análisis crítico de la información (Moreira, 2002; Ricardo, Borjas, Velásquez, Colmenares \& Serje, 2013).

Mantenimiento y seguimiento de los recursos tecnológicos: El soporte técnico es uno de los requerimientos básicos para garantizar la innovación educativa

4 Tecnofilicos y los tecnofóbicos son categorías acuñadas bajo la lógica de "apocalípticos e integrados" propuesta por Umberto Eco (1964). Los primeros son aquellas personas o perspectivas que simpatizan con la instauración de las tecnologías en la vida social, mientras que los segundos son quienes avizoran un futuro nada prometedor para la humanidad debido a la presencia de las tecnologías (Zermeño, Arellano \& Ramírez, 2007, p. 160). 
en TIC, de manera que permita la administración, mantenimiento y reparación del equipamiento dispuesto, que permita la continuidad en integración en todos los temas curriculares (Jiménez \& Gijón, 2016).

Incentivos: Para el MEN, los incentivos ofrecidos a docentes, "pueden generar mayor compromiso en el desempeño de su labor y, de esta forma, mejorar los procesos de enseñanza y de gestión y en consecuencia, de aprendizaje de los estudiantes" (2013b, p. 5).

De esta manera, para aquellos docentes que usen y creen estrategias para la integración de TIC, es prudente contar con un plan que reconozca los logros de los educadores en su liderazgo, que puede iniciar desde el reconocimiento institucional, hasta la implementación de otras tipologías de incentivos como "diferenciaciones salariales, estabilidad laboral, beneficios económicos, desarrollo profesional, infraestructura y materiales educativos adecuados, autonomía y reconocimiento, y mayores grados de responsabilidad” (MEN, 2013b, p. 15), según las características y disponibilidad de la institución educativa.

Se sugiere que esta secuencia sea un proceso cíclico de manera que permita reevaluar y fortalecer cada uno de las etapas, además de ser el garante para desarrollar estrategias permanentes que permitan la incorporación de las TIC de manera efectiva en todos los procesos institucionales comunicativos, de gestión, investigativos, pedagógicos, etc.-.

\section{Consideraciones finales}

La investigación encontró que, a pesar de las políticas que en materia de TIC han propendido en los últimos decenios por fortalecer la conectividad en las zonas apartadas y generar las condiciones para su utilización y el desarrollo de los territorios, la realidad de las instituciones educativas rurales muestra que, aunque se ha invertido en infraestructura, el acceso a internet de forma permanente y de calidad no se ha logrado materializar, aun cuando es considerado por los docentes como una herramienta importante para el desarrollo de las prácticas de aula.

Si bien las instituciones no cuentan con conectividad de manera estable y permanente, y las fallas de energía eléctrica son frecuentes, los docentes han optado por estrategias alternas, como el software off- 
line, material audiovisual proyectado desde los video beams y la carga de los dispositivos como tabletas en los hogares de los profesores para garantizar su disponibilidad en las prácticas de aula.

En cuanto a la razón de estudiantes promedio por computador y tableta, se evidenció que mientras en algunas instituciones la razón es de 2 tabletas por cada 7 estudiantes, en otras hay 1 artefacto por cada 30 niños, siendo amplia la disparidad entre centros educativos, además de reportar baja periodicidad en las acciones destinadas al mantenimiento de los equipos informáticos, haciendo pensar en la necesidad de crear estrategias de capacitación de maestros para la sostenibilidad de los proyectos mediante la preparación de los maestros para hacer frente a las eventualidades que requieren de mantenimiento correctivo y preventivo.

Los docentes de las instituciones rurales encuestadas reportan que, aunque para los centros educativos, las TIC se reconocen en el currículo con un grado considerable de importancia, los recursos tecnológicos con los cuales se cuenta no suplen las necesidades, debido a aspectos de desactualización de equipos, bajo seguimiento a las políticas de Estado y desfase en los proyectos en la relación y adaptación a los contextos.

Así mismo los docentes muestran cierto grado de pesimismo en relación con las cualificaciones ofrecidas por el Estado, que como afirma la Unesco es un malestar general, derivado de la desconexión que existe entre el diseño de dichas capacitaciones y la práctica cotidiana del educador.

Finalmente, con el propósito de sugerir un proceso de consolidación de entornos de aprendizaje que favorezcan la incorporación de TIC, determinado por los maestros y derivado de su experiencia, los docentes concuerdan en que la secuencia para un plan estratégico estaría compuesta de la siguiente manera: primero la planificación, en la cual se han de revisar los aspectos de adaptación curricular y disponibilidad de los maestros; en segunda instancia, la capacitación de maestros como mecanismo de perfeccionamiento y apoyo continuo y contextualizado; seguido de la disponibilidad de recursos tecnológicos y de mantenimiento de los mismos; y para finalizar, con un programa de incentivos que mantenga el interés y el liderazgo de los educadores por la integración de las TIC en su quehacer pedagógico. 


\section{Referencias}

Bacigalupo, C., \& Montaño, V. (2005). Modelo de incorporación de TIC en el proceso de innovación docente para la implementación de un b-learning. Virtual Educa 2005. Recuperado de https://ddd.uab.cat/pub/ $\operatorname{dim} / 16993748$ n11/16993748n11a2.pdf

Barón, L., \& Gómez, R. (julio-diciembre, 2012). De la infraestructura a la apropiación social: panorama sobre las políticas de las tecnologías de la información (TIC) en Colombia. Signo y Pensamiento, XXXI, 34-55.

CAMARDA, P. (2016). Ruralidades, Educación y TIC: Desafíos urgentes para las politicas educativas de Integración de TIC. Buenos Aires: IIPE-Unesco.

Clacso. (2015). Formación docente y pensamiento crítico. Recuperado de: https://www. clacso.org.ar/grupos_trabajo/detalle_gt.php?ficha $=579 \& s=5 \&$ idioma $=$

Claro, M. (2010). La incorporación de tecnologias digitales en educación. Modelos de identificación de buenas prácticas. Santiago de Chile: CEPAL, Comisión Económica para América Latina y el Caribe.

Colombia. (1998). Plan Nacional de Desarrollo Cambio para Construir la Paæ: Bogotá: Departamento Nacional de Planeación.

DNP, MEN., \& SENA. (1999). Compes 3063. Bogotá: Departamento Nacional de Planeación.

DNP. (1994). Documento CONPES 2739-COLCIENCLAS-DNP:UDE. Santafé de Bogotá D.C: República de Colombia.

Felizzola, Y. (2010). Tecnologías de información y comunicación para el desarrollo rural en Colombia. Economía Gestión y Desarrollo, (10), 97-124.

Hernández, U., Gómez, A., \& Montenegro, Y. (2014). Modelo de referencia para la planeación estratégica de TIC focalizada. Revista $S \& T, 12(28)$, 27-51.

IPEE Unesco. (2014). Las nuevas generaciones de mujeres rurales como promotoras del cambio. Recuperado de https://www.buenosaires.iiep.unesco.org/sites/ default/files/MUJERES_RURALES_Q_FINAL.pdf

JimÉnEZ, N., \& Gijón, J. (2016). Las TIC en los países andinos: programas escolares y papel del docente. ENS AYOS, Revista de la Facultad de Educación de Albacete, 31(1), 165-181.

LONDOÑo, F., \& CASTILLO, F. (2012). Planestic: un modelo para formulación de planes. Memorias del XVII Congreso Internacional de Informática Educativa, TISE. Recuperado de http://www.tise.cl/volumen8/TISE2012/19.pdf 
LÓPEZ, J. (2015). S AMR, modelopara integrar las tic en procesos educativos. Recuperado de http://eduteka.icesi.edu.co/articulos/samr

Mattelart, A. (2007). Historia de la sociedad de la información (Trad. G. Multigner,). Barcelona: Paidós.

MEN, MinTIC, SENA., \& Computadores para Educar. (2016). Informe de Gestión de Computadores para Educar 2016. Bogotá.

MEN. (2013a). Competencias TIC para el desarrollo profesional Docente. Bogotá: Ministerio de Educación Nacional.

MEN. (2013b). Hacia la creación y consolidación de un plan de Incentivos para Docentes y Directivos Docentes del sector oficial, con miras al mejoramiento de la calidad de la educación en Colombia. Bogotá: Ministerio de Educación Nacional.

MenchÉn, F. (2009). El maestro creativo: nuevas competencias. Tendencias pedagógicas, (14), 279-290.

MesA, F. (2012). Las Tecnologías de la Información y la Comunicación en la universidad colombiana: Evolución y prospectiva. Revista Historia de la Educación Latinoamericana, 14 (19), 71-90.

Mesa, F., \& Forero, A. (2016). Las TIC en la normativa para los programas de educación superior en Colombia. Praxis \& Saber, 7(14), 91-113. https://doi.org/10.19053/22160159.5219

Ministerio de Comunicaciones. (2000). Decreto 2324. Bogotá: Ministerio de Comunicaciones.

Ministerio de Comunicaciones. (2008). Plan Nacional de Tecnologías de la Información y las Comunicaciones. Bogotá: Ministerio de Comunicaciones.

Morante, F., Mocelin, A., \& Zilles, R. (2006). Capacitación y transferencia tecnológica: Su importancia en la sostenibilidad de los proyectos basados en tecnología solar fotovoltaica. AVERMAS, Avances en Energias Renovables y Medio Ambiente, Edición especial de la Asociación Argentina de Energias Renovables y Ambiente, AS ADES, 10, 1-8.

Moreira, M. (2002). La integración escolar de las nuevas tecnologías. Entre el deseo y la realidad. Revista del Fórum Europeo de Administradores de la Educación, 10(6), 14-18.

ORTIZ, R. (2005). Apropiación social de las tecnologías de la información: ciberciudadanías emergentes. Tecnología y comunicación educativas (TyCE), 41, 19-32. 
Pérez, M., Fandos, M., \& Aguaded, J. (2009). Estudio del modelo Andaluz de implementación de las TIC en los centros escolares: Algunas valoraciones y propuestas. Horizontes Educacionales, 14(2), 49-68.

Ricardo, C., Borjas, M., Velásquez, I., Colmenares, J., \& Serje, A. (2013). Caracterización de la Integración de las TIC en los currículos escolares de las instituciones educativas en la ciudad de Barranquilla. Zona Próxima, (18), 32-45.

SAn Martín, Á. (2009). La escuela enredada. Formas de participación en la sociedad de la Información. Barcelona: Gedisa.

Soto, D., MesA, F., \& Caro, E. (2012). Convergencia digital en la Universidad Colombiana. Del siglo XX al XXI. Revista Historia de la Educación Latinoamericana, 14(19), 265-300.

Soto, J., Franco, M., \& Giraldo, J. (2014). Desarrollo de una metodología para integrar las TIC ( Tecnologías de la Información y la Comunicación) en las IE (Instituciones Educativas) de Montería. Zona Próxima, (21), 33-50.

UnesCo. (2002). Formación docente: un aporte a la discusión. La experiencia de algunos paises. Santiago de Chile.

UnesCo. (2004). Las Tecnologias de la Información y la Comunicación en la formación docente. Guía de planificación. París: Unesco.

VÁsQUEZ, V. (2005). Modelo de incorporación de TIC en el proceso de innovación docente para la implementación de un b-learning. Recuperado de http://e-spacio.uned. es/fez/eserv/bibliuned:19397/n04montano05.pdf

Zermeño, A., Arellano, A., \& Ramírez, V. (2007). El papel de las tecnologías de información y comunicación en la vida cotidiana. Estudios sobre las Culturas Contemporáneas, XIII(25), 152-160. 